\title{
NON-SEROLOGIC TEST FOR COVID-19: HOW TO MANAGE?
}

\author{
Sara Torretta et al. ${ }^{1}$ \\ ${ }^{1}$ Affiliation not available
}

May 11, 2020

\begin{abstract}
Background: Diagnosis of Severe Acute Respiratory Coranavirus-2 (SARS-CoV-2) infection is currently based on Real-Time PCR (RT-PCR) performed on either nasopharyngeal (NPS) or oropharyngeal (OPS) swabs; saliva specimen collection can be used, too. Diagnostic accuracy of these procedures is suboptimal, and some procedural mistakes may account for it.

Methods and results: The video shows how to properly collect secretions from the upper airways for non-serologic diagnosis of COVID-19 by nasopharyngeal swab (NPS), oropharyngeal swab (OPS), and deep saliva collection after throat-cleaning manoeuvre, all performed under videoendoscopic view by a trained ENT examiner.

Conclusions: We recommend to perform NPS after elevation of the tip of the nose in order to reduce the risk of contamination from the nasal vestible, and to let it flow over the floor of the nasal cavity in parallel to the hard palate in order to reach the nasopharynx. Then the tip of the swab should be left in place for few seconds, and then rotated in order to achieve the largest absorption of nasopharyngeal secretions. Regards OPS, gentle anterior tongue depression should be used to avoid swab contamination from the oral cavity during collection of secretions from the posterior pharyngeal wall. These procedural tricks would enhance diagnostic reliability.
\end{abstract}

This manuscript supplements this Operative Techniques video presentation:

Rich media available at https://youtu.be/T6dqzmj2ErM

\section{Author list}

Sara Torretta, ${ }^{1,2}$ MD; Gianvincenzo Zuccotti, ${ }^{3}$ MD; Valentina Cristofaro, ${ }^{1,2}$ MD; Jacopo Ettori, ${ }^{1,2}$ MD; Lorenzo Solimeno, ${ }^{1,2}$ MD; Ludovica Battilocchi, ${ }^{1,2}$ MD; Alessandra D’Onghia, ${ }^{1,2}$ MD; Lorenzo Pignataro, ${ }^{1,2}$ MD; Pasquale Capaccio, ${ }^{1,4}$ MD.

${ }^{1}$ Department of Otolaryngology and Head and Neck Surgery, Fondazione IRCCS Ca' Granda Ospedale Maggiore Policlinico; ${ }^{2}$ Department of Clinical Sciences and Community Health, University of Milan; ${ }^{3}$ Department of Biomedical and Clinical Sciences Luigi Sacco, University of Milan; ${ }^{4}$ Department of Biomedical Surgical Dental Science, University of Milan; Milan, Italy.

\section{Introduction}

Diagnosis of Severe Acute Respiratory Coranavirus-2 (SARS-CoV-2) infection is currently based on RealTime PCR (RT-PCR) performed on either nasopharyngeal (NPS) or oropharyngeal (OPS) swabs; ${ }^{1}$ however, it has been described that a negative NPS or OPS does not rule out coronavirus 2019 (COVID-19), and this could be related to different situations. ${ }^{2}$ Given the fact that SARS-CoV-2 RNA title in the upper respiratory tract peaks between days 7-10 after the clinical onset, a late sample timing could account for a false negative result. $^{2}$ 
It is well known that diagnostic accuracy of NPS and OPS is not so high, being the detection rate of SARS-CoV-2 RNA respectively of $63 \%$ and $32 \% ;^{1}$ therefore, UNITED States Centers for Disease Control and Prevention have recommended the collection of sole upper respiratory NPS. ${ }^{1}$ Reduced detection rate could be related to either inadequacy of sample collection into the nasopharynx (the risk that the collection of secretion is performed into the nasal cavity rather than the nasopharynx is not neglectable, given the incomplete patient cooperation during this unpleasant manoeuvre), ${ }^{3}$ or a limited viral local tropism due to the low expression of ACE-2 receptors in the epithelial cells of the nasopharyngeal/oropharyngeal surface.

Despite these considerations, collection of upper airway secretions by means of NPS/OPS still represents the first line diagnostic modality to test patients and otherwise asymptomatic population for COVID-19, provided that it is early and adequately performed after onset of symptoms. Self-collection of saliva samples has been proved to be an alternative safe, cheap and non-invasive diagnostic mean to confirm SARS-CoV-2 infection. ${ }^{4,5}$

\section{Methods}

We propose a videoclip showing the three main sample collection procedures (i.e.: NPS, OPS, and saliva collection after throat-cleaning manoeuvre) performed under videoendoscopic view by a trained ENT examiner.

\section{Results}

We recommend to perform NPS after elevation of the tip of the nose in order to reduce the risk of contamination from the nasal vestibule (Figure 1), and to let it flow over the floor of the nasal cavity in parallel to the hard palate in order to reach the nasopharynx. Then the tip of the swab should be left in place for few seconds, and then rotated in order to achieve the largest absorption of nasopharyngeal secretions. Regards OPS, gentle anterior tongue depression should be used to avoid swab contamination from the oral cavity during collection of secretions from the posterior pharyngeal wall (Figure 2).

With regards to self-collection of saliva samples, a throat-cleaning manoeuvre would be useful to retrieve infected secretions both descending from the nasopharynx and moving up from the tracheo-bronchial district.

\section{Conclusions}

These procedural tricks would enhance diagnostic reliability, in particular in the case of NPS, given that the risk of collecting secretions from the nasal cavity rather than the nasopharynx is unneglectable, also on the basis of incomplete patient cooperation during this unpleasant manoeuvre.

\section{REFERENCES}

1. Centers for Disease Control and Prevention. Interim Guidelines for Collecting, Handling, and Testing Clinical Specimens from Persons for Coronavirus Disease 2019 (COVID-19). Available at: https://www.cdc.gov/coronavirus/2019-nCoV/lab/guidelines-clinical-specimens.html. Accessed April 16th 2020

2. Winichakoon P, Chaiwarith R, Liwsrisakun C, et al. Negative Nasopharyngeal and Oropharyngeal Swab Does Not Rule Out COVID-19. J Clin Microbiol 2020;26: 00297-00220.

3. Lippi G, Simundic AM, Plebani M. Potential preanalytical and analytical vulnerabilities in the laboratory diagnosis of coronavirus disease 2019 (COVID-19). Clin Chem Lab Med 2020.

4. To KK, Tsang OT, Chik-Yan Yip C, et al. Consistent detection of 2019 novel coronavirus in saliva. Clin Infect Dis 2020;12:ciaa149.

5. To KK, Tsang OT, Leung WS, et al. Temporal profiles of viral load in posterior oropharyngeal saliva samples and serum antibody responses during infection by SARS-CoV-2: an observational cohort study. Lancet Infect Dis 2020;23:S1473-099(20)30196-1. 
